\title{
Research focuses and advance technologies of smart grid in recent years
}

\author{
MEI ShengWei \& CHEN LaiJun* \\ State Key Laboratory of Control and Simulation of Power Systems and Generation Equipments, Department of Electrical Engineering, Tsinghua \\ University, Beijing 100084, China
}

Received April 23, 2012; accepted May 4, 2012

\begin{abstract}
The smart grid is attracting more and more attention in electrical engineering for its promising features. However, huge challenges also exist in the implementations of the smart grid, such as high penetration of renewable energy resources, low carbon electricity and cyber-physical security, etc. In this paper, the latest progress of some research focuses and advance technologies in the development of smart grid is reviewed.
\end{abstract}

smart grid, renewable energy integration, low carbon electricity, cyber-physical security, power system stability, energy storage system, high temperature superconductivity

Citation: $\quad$ Mei S W, Chen L J. Research focuses and advance technologies of smart grid in recent years. Chin Sci Bull, 2012, 57: 2879-2886, doi: 10.1007/s11434$012-5261-5$

The smart grid plays an important role in the response to the energy crisis and global warming challenges and has become a worldwide hot topic in recent years in the field of electrical engineering.

According to the US department of energy, the smart grid is expected to have the following characteristics: capability of self-healing from faults, robustness under cyber and physical attacks, high reliability and power quality, active participation of consumers and accommodation of all generation and storage options [1].

In order to achieve the ultimate goal of the smart grid, researchers have made many efforts to present new theories, techniques and mechanisms to build a secure, economical and environment-friendly power system.

In this context, many new research areas become hot spots of smart grid, including renewable energy integration aiming to mitigate the fossil fuels depletion, low carbon electricity for decreasing the emission of greenhouse gas, cyber-physical security investigation in a highly information based power system, etc.

*Corresponding author (email: chenlaijun@mail.tsinghua.edu.cn)
Moreover, advances in theory and technology have injected new vitality to the traditional electrical engineering research area, including modern control theory and wide area measurement technology for power system stability analysis and control, novel methodology and its application in the design and control of energy storage system, fantastic phenomena and practical implementation in high temperature superconductivity research, etc.

In this paper, a review of some research focuses and advance technologies of smart grid in the last three years is made based on a comprehensive survey covering the most important journals in electrical engineering, such as IEEE Transactions on Power System, IEEE Transactions on Smart Grid, Science China Technological Sciences, etc. The main purpose of this paper is to provide a reference for relative researchers to learn about the latest research progress and find potential research spots.

\section{Renewable energy integration}

With the crisis resulted from fossil fuels depletion and 
global warming intensifies, development and utilization of renewable energy is taken as an important measure to meet this challenge. Among types of renewable energy, wind energy and solar energy are given more and more attention nowadays.

\subsection{Wind energy integration}

Wind energy has the characteristics of randomness and intermittent, which introduce adverse impacts on many aspects when wind power is integrated to power system, such as voltage stability, frequency stability and power quality, etc. A lot of work has been done to overcome these problems brought about by the wind power integration.

The spatiotemporal complementarity of the dispersed wind energy resources in China was investigated in [2]. The results show the feasibility of using the spatiotemporal complementarity of the wind energy resources to smooth power output as well as to improve the performance of wind power forecasting. A dispatch strategy based on fuzzy clustering algorithm was proposed for the short-term schedule problem of a wind farm [3]. Test results show that the dispatch solution obtained in this work can effectively reduce the operation loss with which the grid instruction satisfied.

Wind turbine is one of the key factors that influence the efficiency in the wind power integration. In [4], the energy efficiency of wind turbine was investigated by analyzing the influences of airfoil aerodynamic parameters on wind turbine blade. Theoretical analysis and practical test show that airfoil lift coefficient is the key parameter which has great influence on the energy efficiency of horizontal axis wind turbines. The blade pitch loads for large scale wind turbines was studied from the perspectives of aerodynamic force, centrifugal force and gravity in [5]. The evaluation models for blade pitch loads were established and validated by the case study of a practical 1.2 MW wind turbine. Small wind turbines are widely utilized to produce power in areas without good wind conditions. In [6], a new low Reynolds number airfoil with good aerodynamic characteristics was proposed and used to improve the efficiency of the small horizontal axis wind turbines.

The wind turbine has different inertial response characteristics compared with the conventional synchronous generator. In order to improve the penetration of wind power, auxiliary energy and control services are required, thus greatly increasing the cost of wind power supply.

A time series sampling methodology was proposed to evaluate the impact of high wind power penetrations on the power system over an extended timeframe [7]. Assessment of power system voltage stability under large penetrations of wind generation was discussed in [8]. In this work, the control characteristics of the wind turbine were utilized to improve the voltage stability margin on both of the distribution level and transmission level. The operation of a power system with high wind energy penetration was modeled in a unit commitment problem [9]. The relationship between the reactive power control of variable speed wind power generators and the rotor angle stability of the synchronous generators was investigated [10]. Results show that the reactive power control strategy employed by the wind power generator has a direct impact on the rotor angle of the synchronous generator. In [11], a hybrid excited doubly salient stator-permanent-magnet motor with high efficiency and power density was presented, which has great potential in wind power generation application.

\subsection{Solar energy integration}

The widespread solar energy is an important renewable energy source with the advantages of being sustainable, reusable and environment-friendly. However, many challenges exist in the utilization of the solar energy due to their low efficiency of energy conversion, fluctuation of power output and low density of energy storage.

A high performance dye-sensitized solar cell (DSSC) was proposed by the application of upconversion luminescence in [12]. To extend the photoelectric response range of the DSSC, a flexible DSSC was fabricated in [13]. Test results show that the proposed cell can achieve an efficiency of $1.43 \%$ in light-to-electric energy conversion.

Generally, there is an optimal operating point that the maximum power output can be obtained in the photovoltaic array. Maximum power point tracking (MPPT) is a key issue in improving the energy conversion efficiency of the solar power generation. An optimal operating point tracking strategy based on fuzzy logic control method was proposed to achieve maximum efficiency of the power generation [14]. Test results show that the fuzzy logic control has good performance under the fluctuation of solar radiation.

The photovoltaic generation units have been growing rapidly in recent years. However, the photovoltaic generation is difficult to dispatch for its fluctuation feature. In order to improve the penetration of solar energy, the impact on system voltages of large-scale photovoltaic based DG needs to be studied in depth. A theoretical framework regarding solar energy penetration limits in distribution power systems was established [15]. In this work, the conductor ampacity and voltage rises were identified as the important limiting factors needed to be carefully examined when installing DG. An island partition strategy with low computational complexity was presented based on depth-first dynamic programming algorithm for distribution power system integrated with DG [16].

The economic analysis is another important issue in the development of large-scale photovoltaic power generation. An optimal planning model was proposed for decision makers to maximize the benefit of photovoltaic power generation investments [17]. In this work, the detailed analysis of power generation unit cost was made based on the realistic estimates of demand growth and capacities of generation 
and transmission. Case study of Ontario projects verified the effectiveness of the proposed model.

\section{Low carbon electricity}

Greenhouse gas emission is considered to be the major factor responsible for the global warming. Therefore, low carbon electricity is an important and urgent research area in the development of smart grid.

\section{1 $\mathrm{CO}_{2}$ capture and storage}

The $\mathrm{CO}_{2}$ capture and storage (CCS) technology of power plant is believed to be the most effective technology to fight against the challenge of global warming.

Life cycle assessment (LCA) has been widely applied to various fields related to energy and environment issues. A full life-cycle evaluation of a $2 \times 300$ MW coal-fired power plant was presented in $[18,19]$. In this approach, the resource consumption and the impact of pollutant emissions were investigated, which is useful in making reasonable decisions on the development of low carbon electricity. The environmental impact of power generating systems was investigated in the scope of life cycle inventory analysis based on the matrix method [20]. The proposed method can be used to evaluate the environmental impact of greenhouse gas emissions in power generation.

As an important component of the low carbon electricity, the $\mathrm{CO}_{2}$ capture power plant (CCPP) will become common plant type in the near future. Flexible operation mechanism for carbon capture power plant can not only improve the security of power system but also increase the economic benefit of power plant investment.

A quantitative analysis for the $\mathrm{CO}_{2}$ capture process of the benchmark capture plant was presented in [21]. In this work, plant performance shows great differences between capture plants and non-capture plants. Further, the operation flexibility of typical post combustion CCPP with ancillary services was investigated, and a mathematical model depicting the relationship among capacity penalty, efficiency penalty, net power output, and the net $\mathrm{CO}_{2}$ emission was proposed [22]. Results show that the proposed approach can bring about $20 \%$ operating profits to the CCPP.

\subsection{Power generation expansion planning}

China is one of the countries that are responsible for global warming due to its coal-dominated power supply. Therefore, decarbonisation in power production sector is of great importance. In this context, impacts introduced by low carbon factors on power generation expansion planning (GEP) need to be carefully examined.

A compromised modeling approach for incorporating and formulating those impacts was proposed in [23]. A comprehensive GEP model with a suitably modified objective function and constraints considering the impacts of feed-in tariffs, quota obligation, emission trade, was proposed in [24]. This work provides a powerful tool for decision makers to evaluate the benefits under different scenarios.

Economic and secure benefits are mainly concerned in traditional models for planning interconnection between power systems. A planning model considering the low carbon electricity factors was proposed and applied to the interconnection planning problem in the power grids of South China [25]. Test results show that the low carbon model is rational and comprehensive compared to the traditional model.

In [26], a high pressure ratio transonic compressor for internal combustion engine was proposed, with a $40 \%$ improvement in power density, which can greatly reduce the emission of $\mathrm{CO}_{2}$.

\section{Cyber-physical security}

Information and communication technologies are widely used in the smart grid construction, which make the modern power system as a cyber-physical system. As a critical aspect of the smart grid, the cyber-physical security problem is attracting more and more attention. Researches on cyber-physical security mainly concern the theories and techniques for modeling the system with malicious accesses and attacks, assessment of system vulnerability, intrusion detection and mitigation, etc.

\subsection{Cyber-physical security modeling}

Traditional power system security modeling mainly focused on individual attacks. However, with the help of modern internet and communication technologies, it is possible to launch a coordinate simultaneous attack by geographically distributed groups. It should be noted that, the negative effect of coordinated attacks carried out by highly motivated, sophisticated groups is much greater than the sum of its individual attacks. Therefore, there is an urgent need to develop mathematical tools to model and analyze the coordinated attacks on cyber-physical systems.

A novel Petri Net construction method accounting for sophisticated attacks on the smart grid was presented in [27]. This method allows different security experts to create smaller Petri Net models separately. Thus, the proposed method offers more flexibility than traditional ones based on attack trees.

In [28], a novel cyber-based dynamical model considering the cyber technologies employed in the power system was proposed. Results show that the model can be used to obtain the full observability without requiring local observability of individual system components. It should be noted 
that, the proposed model is also very effective in distributed decision making for its network structure-preserving properties.

\subsection{Cyber-physical security assessment and mitigation}

Although the control centers of power system are usually highly secured, the cyber-physical system is vulnerable due to the potential attacks that are launched from distributed areas and different means. Therefore, there is a strong need to assess the security of the overall cyber-physical system and investigate corresponding countermeasures.

An attack-tree based method was proposed to evaluate the vulnerabilities of cyber-physical system under three types of malicious attacks: attack upon the system, attack by the system and attack through the system [29]. A vulnerability assessment framework embedded with the firewall and password models was proposed to evaluate the security of SCADA systems in three levels: system, scenarios, and access points [30].

In [31], a probabilistic cyber security index for risk evaluation of cyber-physical system was proposed. In this work, an integrated SCADA model and a power flow program were used to determine the risk measure. An anomaly inference method considering temporal anomalies was developed to monitor the cyber security of the substations [32]. The approach is a useful tool for early detection of cyber-intrusions that are likely to cause significant damages to the power grid.

\section{Advance theory and technology for power system stability}

In order to meet the growing demand for energy consumption, generators with huge capacity are widely deployed in the power system. Consequently, the transient stability problem is becoming more and more serious due to the increasingly bulk power exchanges over long distance. Therefore, effective analysis methods and control measures are required to guarantee the stability of the power system.

\subsection{Advances in power system stability analysis}

The stability region estimation of power system is an interesting topic in the transient stability analysis and the dynamic security assessment. In [33], a method considering the output uncertainty of the wind farms was proposed to determine the local boundaries of voltage stability region for power system with wind power integration. The expansion of the estimated stability region is an important issue in the stability analysis. A mathematical foundation for the expansion methods of the estimated stability region was presented for developing algorithms to enlarge or compressing the estimated stability region [34]. This work pointed out that the expansion of the estimated stability region can be directly obtained from the diffeomorphism map.

The calculation of the unstable equilibrium point (UEP) plays an important role in stability region estimation of power system. Generally, the stability of the most commonly used induction motors greatly affects the transient voltage stability of power system. An approach based on continuation method for computing the UEP of the power system with induction motors was proposed in [35]. In this work, a parameter was introduced into the state equation to represent the mechanical torques of motors.

A method based on a quadratic form local input-to-state stability (LISS)-Lyapunov function was presented to analyze the LISS properties in [36]. Numerical simulation tests on power system with power electronics validated the effectiveness of the proposed method. A multi-level multi-area hybrid controller was developed for the automatic voltage control in Northeast China grid [37]. This work successfully investigated and solved the following three hard problems: the coordination of AVC systems, the closed-loop voltage control method for multi-objective optimization and the high quality data for close-loop voltage control.

The load model is of great importance to the analysis of power system stability. A synthesis load model based on the combination of the component and measurement was proposed in [38]. In this work, the identifiability and identification of the synthesis load model were discussed. A novel evolving model considering the property of power grids was proposed based on the Barabási-Albert (BA) model [39], in which the simulation results show that the characteristic of real power network is between scale-free network and totally uncertain network.

The damping torque of the voltage source converter (VSC) on power system oscillations was carefully examined in [40]. Analysis reveals that the AC voltage control of VSC has little impact on the damping of power system oscillations, while the DC voltage control of VSC shows different damping characteristics under different load conditions. A novel approach integrated with the improved empirical mode decomposition and signal energy algorithm was presented to investigate the dominant oscillation mode [41]. In this work, the low frequency oscillation problem was analyzed from the energy view.

An updating preconditioner in iterative method was presented to speed up the time domain on-line security assessment of large scale power system [42]. Case study on the practical systems of China shows that the proposed algorithm can reduce $9 \%-61 \%$ iteration count compared with the dishonest preconditioner algorithm. Dynamic equivalence is an effective approach to simplify the analysis and control of large scale power system. A dynamic equivalent method with consideration of dynamic characteristics of motor loads was proposed for interconnected power systems [43]. Test results show that the proposed method can significantly reduce the system size while maintain the domi- 
nant features of the original system.

Wide area measurement system (WAMS) can be used to measure and record the operation states of power systems continuously. Therefore, it is a powerful tool to detect and analyze low-frequency oscillation. Based on the frequency information provided by the WAMS, the time varying characteristics of a low frequency oscillation of the Guizhou Power Grid was investigated in [44]. A wide-area nonlinear robust controller was proposed for the voltage regulation of the multi-machine power systems [45]. The controller shows good performance in the different system operation parameters and states.

\subsection{Advances in power system stability control}

Modern control theory and technology are of great importance in improving the power system stability. Among them, power system stabilizer (PSS) is considered to be the most cost-effective way to solve the low frequency oscillation problem.

The classical design theory and modern linear optimal feedback controller design theory for PSS are thoroughly investigated in both the frequency domain and the time domain [46]. The nature of the PSS work principle was discussed in this work. Based on extended quasi-steady-state (QSS) model including slow inter-area oscillations, a PSS design approach that does not require full system representation was proposed to eliminate the oscillations problem in a two-area test system with 11 buses and 4 generators [47].

A PSS design method for interconnected multi-machine power systems was proposed in [48]. Compared with traditional design methods, all PSS design parameters in this method were calculated using only the local measurements thus no information of external equivalent impedances and bus voltage were required. A coordinated method within the frequency-domain for the design of PSS and automatic voltage regulator (AVR) in a tightly coupled multi-machine system was proposed in [49]. Both the system transient stability and the system oscillation stability were considered in this work. Test results show the feasibility of decoupling the design of PSSs and AVRs.

Some difficulties are encountered when PSS and FACTS devices are used to damp the inter-area modes oscillation, due to the limited adjustable capacity of generator under PSS control and the regulation capability of FACTS supplementary damping control. With the help of the widely used WAMS, a delicate method for the design of multiple HVDC damping controller was presented to mitigate the inter-area modes oscillation in [50], which can significantly improve the damping performance.

\section{Energy storage system}

Great challenges have been brought to the operation of smart grid by the renewable energy, especially from wind energy and solar energy, due to their variability and nondispatchable features. In this context, the energy storage system (ESS) is an effective solution to maintain the balance between energy production and consumption and to provide high quality and reliable power supply for the customers, by means of storing energy at the time of surplus and releasing energy when needed.

\subsection{Energy storage system design}

Sizing is one of the key issues that need to be considered in the design of the ESS. In [51], a novel methodology was proposed to evaluate the maximum feasible size of the ESS. Instead of using traditional analysis models, such as production cost model and comprehensive storage model, the maximum size was determined by identifying different cycling components of the balancing power. Based on the cost-benefit analysis, a new method was proposed to determine the optimal size of ESS for both islanded and grid-connected modes of micro grid [52]. The optimal solution was obtained by formulating the problem in the form of mixed integer linear programming.

The allocation of the ESS has an important impact on the operation performance of the power system. Therefore, in-depth researches on optimal allocation methods for ESS are required. In [53], the allocation optimization of the ESS in distribution systems with high percent wind energy integration was investigated. In this work, a method based on OPF was proposed to maximize the profit of both the DG producers and the customers. A novel approach based on the matrix real-coded genetic algorithm (MRCGA) was presented to solve the optimal allocation and economic operation problem of ESS in micro grids [54]. In this approach, the optimal number and type of DG and energy storage devices were determined with the consideration of operational and economical relationship between different energy storage devices.

\subsection{Energy storage system control}

The control strategy of ESS has a direct impact on the performance of power system, especially in the power system integrated with large-scale wind power.

Four types of control strategy including simple control, fuzzy control, simple artificial neural network (ANN) control and advanced ANN control for a zinc-bromine flow battery based ESS were investigated and compared in [55]. Test results show that through efficient control strategy of the ESS, the deviation between actual output and predicted output of the wind farm can be controlled within $4 \%$ for $90 \%$ of the situations.

Battery and supercapacitor are important components of the hybrid energy storage system (HESS). Compared with the supercapacitor, the battery owns the advantage of high 
energy density but has the disadvantage of low power density. In [56], a control scheme for the energy management optimization of a HESS was proposed to minimize both of the current fluctuation of the battery and the energy loss of supercapacitor. In this work, the best control scheme was obtained by formulating the energy management of the HESS as an optimization problem. A supercapacitor-battery energy storage system with high performance was proposed [57]. In this approach, the working principle of this type of ESS was studied.

\section{High temperature superconductivity}

High temperature superconductivity (HTS) means zero resistance at the superconducting state. This property creates many fantastic phenomena and applications.

\subsection{Characteristic of HTS}

A new phenomenon named "super-proximity-effect" was discovered in the experiments with multilayer HTS. Experiments show that alternating current mainly flows in the inner and outer layers of the cable. This phenomenon has significant importance to the design of the multilayer superconductor that used in power electricity transmission [58].

The current distribution in a hybrid conductor consisting of low temperature superconductivity (LTS) and HTS was investigated by numerical simulation method based on the power-law models [59]. Results show that the current in the HTS increases with the temperature arise as long as the current is under the critical value.

In [60], methods based on statistical analysis were proposed to investigate the inhomogeneity of critical current and index $n$ value on HTS tapes, which provide a useful approach for the design of HTS application. The A15 phase composition and critical temperature of superconducting strands were investigated in [61].

\subsection{Application of HTS}

The HTS is one of the most promising technologies that will be widely utilized in the smart grid for its unique characteristics.

Energy storage is an important application area of superconductor, which has many advantages, such as high cyclic efficiency, deep discharge and recharge ability, fast response capability and good balance between power density and energy density. In [62], a design of a superconducting magnetic energy storage (SMES) coil was proposed to reach the maximum energy storage in a coil made by conductors with a length of $100 \mathrm{~m}$.

The wind turbine integrated with HTS technology shows great performance in increasing the stability of the wind power output while decreasing the volume and weight of the generators. A conceptual design of a $100 \mathrm{~kW}$ wind turbine generator using HTS was proposed in [63] and investigated for the feasibility of $10 \mathrm{MW}$ wind turbine generators in [64]. The characteristics of a 10 MW HTS wind turbine generator under different operation conditions were carefully examined through numerical simulations based on finite element method [65].

\section{Conclusions}

This paper makes a brief review on the recent progress of important fields in the smart grid. Six hot research topics are selected and discussed based on the investigations of the latest authoritative literatures.

It should be noted that this paper only covers a small number of attractive aspects of smart grid. Opportunities and challenges coexist in the implementation of smart grid and further researches are required to promote the development of the future power system.

This work was supported by the National Natural Science Foundation of China (50977047) and the National Science Foundation for Post-doctoral Scientists of China (20110490351).

1 Hassan R, Radman G. Survey on smart grid. In: Proceedings of the IEEE SoutheastCon. North Carolina: IEEE, 18-21 March 2010. 210213

2 Liu Y, Xiao L Y, Wang H F, et al. Investigation on the spatiotemporal complementarity of wind energy resources in China. Sci China Technol Sci, 2012, 55: 725-734

3 Liu J Z, Liu Y, Zeng D L, et al. Optimal short-term load dispatch strategy in wind farm. Sci China Technol Sci, 2012, 55: 1140-1145

4 Huang C W, Yang K, Liu Q, et al. A study on performance influences of airfoil aerodynamic parameters and evaluation indicators for the roughness sensitivity on wind turbine blade. Sci China Technol Sci, 2011, 54: 2993-2998

5 Dai J C, Hu Y P, Liu D S, et al. Calculation and characteristics analysis of blade pitch loads for large scale wind turbines. Sci China Technol Sci, 2010, 53: 1356-1363

6 Wata J, Faizal M, Talu B, et al. Studies on a low reynolds number airfoil for small wind turbine applications. Sci China Technol Sci, 2011, 54: 1684-1688

7 Doherty R, Mullane A, Nolan G, et al. An assessment of the impact of wind generation on system frequency control. IEEE T Power Syst, 2010, 25: 452-460

8 Vittal E, O’Malley M, Keane A. A steady-state voltage stability analysis of power systems with high penetrations of wind. IEEE $\mathrm{T}$ Power Syst, 2010, 25: 433-442

9 Dietrich K, Latorre J M, Olmos L, et al. Demand response in an isolated system with high wind integration. IEEE T Power Syst, 2012, 27: 20-29

10 Vittal E, O'Malley M, Keane A. Rotor angle stability with high penetrations of wind generation. IEEE T Power Syst, 2012, 27: 353-362

11 Zhu X Y, Cheng M. Design, analysis and control of hybrid excited doubly salient stator-permanent-magnet motor. Sci China Technol Sci, 2010, 53: 188-199

12 Xie G X, Lin J M, Wu J H, et al. Application of upconversion luminescence in dye-sensitized solar cells. Chin Sci Bull, 2011, 56: 301-308 
13 Yue G T, Wu J H, Xiao Y M, et al. Flexible dye-sensitized solar cell based on PCBM/P3HT heterojunction. Chin Sci Bull, 2011, 56: 325-330

14 Wang C C, Wu M C, Ou S Y, et al. Analysis and research on maximum power point tracking of photovoltaic array with fuzzy logic control and three-point weight comparison method. Sci China Technol Sci, 2010, 53: 2183-2189

15 Shayani R A, de Oliveira M A G. Photovoltaic generation penetration limits in radial distribution systems. IEEE T Power Syst, 2011, 26: 1625-1631

16 Wang X D, Lin J K. Island partition of the distribution system with distributed generation. Sci China Technol Sci, 2010, 53: 3061-3071

17 Muneer W, Bhattacharya K, Canizares C A. Large-scale solar PV investment models, tools, and analysis: The Ontario case. IEEE T Power Syst, 2011, 26: 2547-2555

18 Wang Y, Zhang J Y, Zhao Y C, et al. Exergy life cycle assessment model of " $\mathrm{CO}_{2}$ zero-emission" energy system and application. Sci China Technol Sci, 2011, 54: 3296-3303

19 Wang Y, Zhao Y C, Zhang J Y, et al. Technical-economic evaluation of $\mathrm{O}_{2} / \mathrm{CO}_{2}$ recycle combustion power plant based on life-cycle. Sci China Technol Sci, 2010, 53: 3284-3293

20 Oshima K, Uchiyama Y. Performance analysis for power generating system by using matrix method. Sci China Technol Sci, 2011, 54: 1689-1696

21 Chen Q X, Kang C Q, Xia Q. Modeling flexible operation mechanism of $\mathrm{CO}_{2}$ capture power plant and its effects on power-system operation. IEEE T Energy Conver, 2010, 25: 853-861

22 Chen Q X, Kang C Q, Xia Q, et al. Optimal flexible operation of a $\mathrm{CO}_{2}$ capture power plant in a combined energy and carbon emission market. IEEE T Power Syst, 2012, (99): 1-8

23 Chen Q X, Kang C Q, Xia Q, et al. Power generation expansion planning model towards low-carbon economy and its application in China. IEEE T Power Syst, 2010, 25: 1117-1125

24 Careri F, Genesi C, Marannino P, et al. Generation expansion planning in the age of green economy. IEEE T Power Syst, 2011, 26: 2214-2223

25 Wu Y W, Lou S H, Lu S Y. A model for power system interconnection planning under low-carbon economy with $\mathrm{CO}_{2}$ emission constraints. IEEE T Sust Energy, 2011, 2: 205-214

26 Zheng X Q, Zhang Y J, Yang M Y. Research and development on transonic compressor of high pressure ratio turbocharger for vehicle internal combustion engines. Sci China Technol Sci, 2010, 53: 1817-1823

27 Chen T M, Sanchez-Aarnoutse J C, Buford J. Petri net modeling of cyber-physical attacks on smart grid. IEEE T Smart Grid, 2011, 2: 741-749

28 Marija D, Xie L, Usman A, et al. Modeling of future cyber-physical energy systems for distributed sensing and control. IEEE T Syst Man Cy A, 2010, 40: 825-838

29 Ten C W, Manimaran G, Liu C C. Cybersecurity for critical infrastructures: Attack and defense modeling. IEEE T Syst Man Cy A, 2010, 40: 853-865

30 Ten C W, Liu C C, Manimaran G. Vulnerability assessment of cybersecurity for SCADA systems. IEEE T Power Syst, 2008, 23: 1836-1846

31 Liu C C, Ten C W, Manimaran G. Cybersecurity of SCADA systems: Vulnerability assessment and mitigation. In: Power Systems Conference and Exposition, PSCE'09. Dublin, Ireland: IEEE, 15-18 March 2009. 1-3

32 Ten C W, Hong J, Liu C C. Anomaly detection for cybersecurity of the substations. IEEE T Smart Grid, 2011, 2: 865-873

33 Mu Y F, Jia H J. An approach to determining the local boundaries of voltage stability region with wind farms in power injection space. Sci China Technol Sci, 2010, 53: 3232-3240

34 Liu F, Wei W, Mei S W. On expansion of estimated stability region: Theory, methodology, and application to power systems. Sci China Technol Sci, 2011, 54: 1394-1406
35 Chen L, Xu F, Min Y. New method for computing unstable equilibrium points of power systems with induction motors. Sci China Technol Sci, 2010, 53: 881-885

$36 \mathrm{Wu} \mathrm{S} \mathrm{Y}$, Mei S W, Zhang X M. Estimation of LISS (local input-to-state stability) properties for nonlinear systems. Sci China Technol Sci, 2010, 53: 909-917

37 Zhang X M, Mei S W, Su X Y, et al. Multi-level multi-area hybrid automatic voltage control system and its trial operation in northeast China grid. Sci China Technol Sci, 2011, 54: 2501-2505

$38 \mathrm{Ju} \mathrm{P}$, Jin Y Q, Chen Q, et al. Identifiability and identification of a synthesis load model. Sci China Technol Sci, 2010, 53: 461-468

39 Liu X, Liu T Q, Li X Y. A novel evolving model for power grids. Sci China Technol Sci, 2010, 53: 2862-2866

40 Du W J, Wang H F, Cheng S J, et al. Effect of embedded voltage source converter on power system oscillation damping. Sci China Technol Sci, 2010, 53: 892-901

41 Yang D C, Rehtanz C, Li Y, et al. A novel method for analyzing dominant oscillation mode based on improved emd and signal energy algorithm. Sci China Technol Sci, 2011, 54: 2493-2500

42 Wang K, Xue W, Lin H X, et al. Updating preconditioner for iterative method in time domain simulation of power systems. Sci China Technol Sci, 2011, 54: 1024-1034

43 Zhou H Q, Ju P, Yang H, et al. Dynamic equivalent method of interconnected power systems with consideration of motor loads. Sci China Technol Sci, 2010, 53: 902-908

44 Yuan Y, Sun Y Z, Cheng L, et al. Power system low frequency oscillation monitoring and analysis based on multi-signal online identification. Sci China Technol Sci, 2010, 53: 2589-2596

45 Ruan Y, Yuan R X. Wide-area nonlinear robust voltage control strategy for multi-machine power systems. Sci China Technol Sci, 2012, 55: 1107-1117

46 Ma J, Wang H J, Zhang P. Renewed investigation on power system stabilizer design. Sci China Technol Sci, 2011, 54: 2687-2693

47 Vournas C D, Mantzaris J C. Application of QSS modeling to stabilizer design for interarea oscillations. IEEE T Power Syst, 2010, 25 : 1910-1917

48 Gurrala G, Sen I. Power system stabilizers design for interconnected power systems. IEEE T Power Syst, 2010, 25: 1042-1051

49 Dysko A, Leithead W E, O'Reilly J. Enhanced power system stability by coordinated PSS design. IEEE T Power Syst, 2010, 25: 413422

50 Wang J J, Fu C, Zhang Y. Design of WAMS-based multiple HVDC damping control system. IEEE T Smart Grid, 2011, 2: 363-374

51 Makarov Y V, Du P W, Kintner-Meyer M C W, et al. Sizing energy storage to accommodate high penetration of variable energy resources. IEEE T Sust Energ, 2012, 3: 34-40

52 Chen S X, Gooi H B, Wang M Q. Sizing of energy storage for microgrids. IEEE T Smart Grid, 2012, 3: 142-151

53 Atwa Y M, El-Saadany E F. Optimal allocation of ESS in distribution systems with a high penetration of wind energy. IEEE T Power Syst, 2010, 25: 1815-1822

54 Chen C S, Duan S X, Cai T, et al. Optimal allocation and economic analysis of energy storage system in microgrids. IEEE $\mathrm{T}$ Power Electr, 2011, 26: 2762-2773

55 Brekken T K A, Yokochi A, von Jouanne A, et al. Optimal energy storage sizing and control for wind power applications. IEEE T Sust Energy, 2011, 2: 69-77

56 Mid-Eum C, Seong-Woo K, Seung-Woo S. Energy management optimization in a battery/supercapacitor hybrid energy storage system. IEEE T Smart Grid, 2012, 3: 463-472

57 Guo W, Xin S, Ji M B, et al. Supercapacitor-battery hybrid energy storage devices from an aqueous nitroxide radical active material. Chin Sci Bull, 2011, 56: 2433-2436

58 Xin Y, Gong W Z, Zhang J Y. A nontrivial factor in determining current distribution in an ac HTS cable-proximity effect. Sci China Technol Sci, 2010, 53: 922-928

59 Guan X J, Wang Y S, Zhou W W. Current distribution and stability 
of LTS/HTS hybrid superconducting conductors. Sci China Technol Sci, 2010, 53: 2000-2007

60 Wang Y S, Guan X J, Zhang H Y, et al. Progress in inhomogeneity of critical current and index $n$ value measurements on HTS tapes using contact-free method. Sci China Technol Sci, 2010, 53: 2239-2246

61 Sulpice A, Soubeyroux J L, Verwaerde C, et al. Representation of A15 composition and $\mathrm{Tc}$ in internal-Sn $\mathrm{Nb}_{3} \mathrm{Sn}$ superconducting strands. Sci China Technol Sci, 2010, 53: 3020-3026

62 Yuan W J, Xian W, Ainslie M, et al. Design and test of a superconducting magnetic energy storage (SMES) coil. IEEE T Appl Super- con, 2010, 20: 1379-1382

63 Li X H, Han L, Yang X L, et al. Design and model test of the racetrack excitation coil in a novel high temperature superconducting generator. IEEE T Appl Supercon, 2010, 20: 1081-1084

64 Li X H, Zhou Y G, Han L, et al. Design of a high temperature superconducting generator for wind power applications. IEEE T Appl Supercon, 2011, 21: 1155-1158

65 Fukui S, Ogawa J, Sato T, et al. Study of $10 \mathrm{mw}$-class wind turbine synchronous generators with HTS field windings. IEEE T Appl Supercon, 2011, 21: 1151-1154

Open Access This article is distributed under the terms of the Creative Commons Attribution License which permits any use, distribution, and reproduction in any medium, provided the original author(s) and source are credited. 